\title{
The optimum decision rules for the oddity task
}

\author{
NIEK J. VERSFELD \\ TNO Human Factors Research Institute, Soesterberg, The Netherlands \\ and \\ HUANPING DAI and DAVID M. GREEN \\ University of Florida, Gainesville, Florida
}

\begin{abstract}
This paper presents the optimum decision rule for an $m$-interval oddity task in which $m-1$ intervals contain the same signal and one is different or odd. The optimum decision rule depends on the degree of correlation among observations. The present approach unifies the different strategies that occur with "roved" or "fixed" experiments (Macmillan \& Creelman, 1991, p. 147). It is shown that the commonly used decision rule for an $m$-interval oddity task corresponds to the special case of highly correlated observations. However, as is also true for the same-different paradigm, there exists a different optimum decision rule when the observations are independent. The relation between the probability of a correct response and $d^{\prime}$ is derived for the three-interval oddity task. Tables are presented of this relation for the three-, four-, and five-interval oddity task. Finally, an experimental method is proposed that allows one to determine the decision rule used by the observer in an oddity experiment.
\end{abstract}

The oddity task provides a means of measuring the observer's ability to discriminate between different stimuli. In the most general form, $m$ stimuli are presented on each trial and there are $m$ response alternatives. Despite the number of response alternatives, there are actually only two stimuli. On any single trial, $m-1$ of the stimuli are the same; only one stimulus is different, the odd stimulus. The observer's task is to select the alternative with the odd stimulus. A frequent argument given for the use of this procedure is that it does not force the observer to distinguish between the two stimuli on some predetermined sensory dimension; rather, it offers the observer the freedom to make the distinction on whatever basis is most convenient. Therefore, when the stimuli are complex - as is often the case in, for example, food research - the oddity task is frequently used to assess the subject's ability to discriminate between different food alternatives.

Forced-choice paradigms differ in the degree and extent to which the specific stimulus alternatives must be labeled or categorized. Without prior knowledge of the stimulus alternatives, it is often difficult to label the stimuli without considerable practice and training. It may even be impossible if the stimuli are not fixed, but vary over trials. To take a concrete example, suppose the task is to select from a pair of sinusoids the one with the higher pitch. One sound has frequency $f-\Delta f$, whereas the other sound has frequency $f+\Delta f$. Let us call $f$ the standard frequency. In a one-interval, forced-choice paradigm, a single sinusoid is

The authors gratefully acknowledge Todd Maddox, Sandy MacRae, and an anonymous reviewer for their valuable comments, and for their suggestions for improving the readability of the manuscript. Correspondence should be addressed to N. J. Versfeld, TNO Human Factors Research Institute, P.O. Box 23, 3769 ZG Soesterberg, The Netherlands. presented. Even if $f$ remained constant over trials ("fixed standard"), it would be difficult to discriminate between the two alternatives unless the listener had near perfect pitch. It would be impossible if $f$ changed randomly from trial to trial ("roving standard"). On the other hand, a threealternative oddity task should require little training, because, for example, the odd stimulus would be presented with frequency $f+\Delta f$, and the other two stimuli would be presented with frequency $f-\Delta f$. Even if $f$ were random, one can imagine the listener performing well above chance and quickly learning the essential stimulus dimension of the discrimination task.

The $m$-interval oddity task should not be confused with the (in psychoacoustic research more common) $m$-alternative forced-choice ( $m$ AFC) task. In both tasks, the observer is presented $m$ stimulus intervals, of which one is different from the other $m-1$ intervals. Suppose that the task is to discriminate between Stimulus $A$ and $B$. In the $m \mathrm{AFC}$ task, each and every trial contains $m-1$ intervals with Stimulus $A$, and the odd interval always contains Stimulus $B$. Thus, for the $m \mathrm{AFC}$ task we have in total $m$ possible sequences of $A$ and $B(B A A A \ldots A A, A B A A \ldots A A$, $A A B A \ldots A A$, etc.). In the oddity task, on the other hand, in one trial the odd interval may contain Stimulus $A$, but in the next one the odd interval may well contain $B$. Thus, here we have in total $2 m$ possible orderings of $A$ and $B$ (BAAA .. AAA, $A B A A \ldots A A$, etc., but also $A B B B \ldots B B$, $B A B B \ldots B B$, etc.). Also, the instruction to the subject is different: To return to our example above, in an $m \mathrm{AFC}$ paradigm the subject's task is to indicate that interval containing the, say, highest pitch, whereas his/her task in the oddity paradigm is to pick the interval that contains the $o d d$ pitch. The differences between the $m$ AFC task and the oddity task have, for $m=3$, been discussed extensively by Frijters (1979a, 1979b). 
As we discuss in this paper, the important difference between the fixed standard and the roving standard task is the degree of statistical correlation found among the observations in such tasks. With a fixed standard, the observations may have zero or little correlation over trials. When the standard changes widely from trial to trial, the observations will be strongly correlated, because the difference between the signal and the standard ( $\Delta f$ in our example) is small in comparison with variations in the standard $(f)$. In most tasks, the value of this correlation is monotonic with the amount or range over which the standard changes over trials. Optimum decision rules for the $m$-alternative oddity task depend on the amount of this correlation and are derived. (Another paradigm in which the shape of the psychometric function depends on the amount of correlation is the so-called same-different paradigm. It is discussed in a companion paper by Dai, Versfeld, \& Green [1996], which may serve the reader as an introduction to the present paper.) For $m=3$, an analytical expression is derived for the expected psychometric function, the relation between the probability of a correct response $P(c)$ and $d^{\prime}$. Tables of $P(c)$ versus $d^{\prime}$ are presented for $m=3,4$, and 5 when the correlation among observations is zero and unity. Finally, an experimental procedure is proposed that enables one to determine what decision rule is actually used by the observer in an experimental oddity task.

\section{SIGNAL DETECTION THEORY}

Signal detection theory provides a framework in which a relationship can be established between the probability of a correct response $P(c)$ in a given detection task and $d^{\prime}$, a parameter that represents the subject's ability to separate Stimulus $A$ and Stimulus $B$. Making such predictions requires several assumptions. The most important of them are summarized in Green and Swets (1988), Frijters (1979b), and Macmillan and Creelman (1991). They are as follows:

1. The presentation of a stimulus $(A$ or $B$ ) results in a number $\xi$ on the decision-axis $X$, and $\xi$ is a realization of a stochastic process, caused by the presentation of the stimulus. The expected value of $\xi$ given Stimulus $A$ is denoted by $\mu_{A}=E[\xi \mid A]$. Likewise, $\mu_{B}=E[\xi \mid B]$. A sequence of $m$ presentations, forming a trial $s$, results in a vector $\xi$ in $m$-dimensional decision space.

2. $\mathbf{x}$ is a random variable with a multivariate Gaussian probability density function $p d f_{s}(\mathbf{x})$, with expected value $\boldsymbol{\mu}_{s}$ and covariance matrix $\Sigma^{2}$. The density function of $\mathbf{x}$ thus becomes

$$
\begin{aligned}
p d f_{s}(\mathbf{x})= & \frac{1}{\sqrt{(2 \pi)^{m}\left|\Sigma^{2}\right|}} \\
& \exp \left[-\frac{1}{2}\left(\mathbf{x}-\boldsymbol{\mu}_{s}\right)^{T} \Sigma^{-2}\left(\mathbf{x}-\boldsymbol{\mu}_{s}\right)\right],
\end{aligned}
$$

where $T$ stands for transpose, $\Sigma^{-2}$ is the inverse matrix, and $\left|\Sigma^{2}\right|$ the determinant of $\Sigma^{2}$

$$
\Sigma^{2}=\left[\begin{array}{cccccc}
\sigma^{2}+\sigma_{R}^{2} & \sigma_{R}^{2} & \cdot & \cdot & \cdot & \sigma_{R}^{2} \\
\sigma_{R}^{2} & \sigma^{2}+\sigma_{R}^{2} & & & & \cdot \\
\cdot & & \cdot & & & \cdot \\
\cdot & & & \cdot & & \cdot \\
\cdot & & & & \sigma^{2}+\sigma_{R}^{2} & \sigma_{R}^{2} \\
\sigma_{R}^{2} & \cdot & . & . & \sigma_{R}^{2} & \sigma^{2}+\sigma_{R}^{2}
\end{array}\right]
$$

The observations may be correlated-due, for example, to a roving-standard experiment-which introduces a common variance $\sigma_{R}^{2} . \sigma^{2}$ is the variance associated with the noisy perception of a stimulus (internal noise) and is assumed to be the same for Stimuli $A$ and $B . \sigma_{R}^{2}$ is the common internal variance caused by the use of a roving-standard paradigm. Hence, the variance in an observation $\xi_{i}$ (by presentation of $A$ or $B$ ) is given by $\operatorname{VAR}\left[\xi_{i}\right]=\sigma^{2}+\sigma_{R}^{2}$ (which are in fact the diagonal elements of $\Sigma^{2}$ ) and is the same for all $i$. The covariance between observations $\xi_{i}$ and $\xi_{j}$ is the same for each pair of observations and is given by $\operatorname{COV}\left[\xi_{i}, \xi_{j}\right]=\sigma_{R}^{2}$ (which are the off-diagonal elements of $\Sigma^{2}$ ). Because of the special form of $\Sigma^{2}$, the correlation coefficient thus always has the same value, and it is given by

$$
\rho=\frac{\sigma_{R}^{2}}{\sigma^{2}+\sigma_{R}^{2}} .
$$

\section{DERIVATION OF THE DECISION RULES FOR THE $M$-INTERVAL ODDITY TASK}

Suppose that a particular sequence $s$ (the $m$ presentations that constitute a trial) has been presented. This gives rise to the vector $\xi$, and the listener can calculate the likelihood that $\boldsymbol{\xi}$ came from a sequence with the odd stimulus in the $i$ th interval, relative to the likelihood that it came from another sequence with the odd stimulus in another interval, say the $k$ th $(k \neq i)$. The likelihood ratio $\mathcal{L}_{i, k}(\xi)$ then is

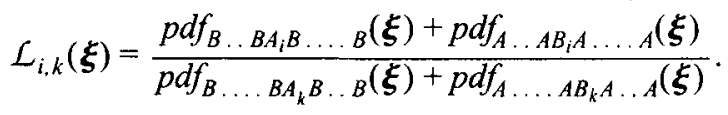

The two terms in the numerator and the denominator arise because the odd interval may comprise Stimulus $A$ or Stimulus $B .^{1}$ The density functions are given in Equation 1, so $\mathcal{L}_{i, k}(\xi)$ can be calculated (Appendix A) and is given by

$$
\mathcal{L}_{i, k}(\boldsymbol{\xi})=\frac{\cosh \left[\frac{d^{\prime}}{2 m \sigma} \sum_{j=1}^{m}\left\{2\left(\xi_{j}-\xi_{i}\right)+(m-2) \varepsilon^{2}\left(\xi_{j}-\mu\right)\right\}\right]}{\cosh \left[\frac{d^{\prime}}{2 m \sigma} \sum_{j=1}^{m}\left\{2\left(\xi_{j}-\xi_{k}\right)+(m-2) \varepsilon^{2}\left(\xi_{j}-\mu\right)\right\}\right]},
$$

where

$$
\begin{aligned}
\cosh (z) & =\frac{e^{-z}+e^{z}}{2}, \\
\varepsilon^{2} & =\frac{\sigma^{2}}{\sigma^{2}+m \sigma_{R}^{2}}=\frac{1-\rho}{1+(m-1) \rho},
\end{aligned}
$$




$$
\mu=\frac{\mu_{A}+\mu_{B}}{2},
$$

and

$$
d^{\prime}=\frac{\mu_{B}-\mu_{A}}{\sigma}
$$

$\varepsilon^{2}$, a measure for the correlation $\rho$ between the observations, is introduced for notational convenience. If observations are uncorrelated $(\rho=0)$, then $\varepsilon^{2}=1$. If the observations are highly correlated $(\rho \approx 1)$, then $\varepsilon^{2} \approx 0$, as can be deduced from Equation 7. $d^{\prime}$ is the modeled observer's sensitivity for the difference between Stimulus $A$ and Stimulus $B$, and $\mu$ is simply the mean of $\mu_{A}$ and $\mu_{B}$.

Equation 5 can simply be rewritten as

$$
\mathcal{L}_{i, k}(\xi)=\frac{\cosh \left[\frac{d^{\prime}}{\sigma}\left(\xi_{i}-\frac{(m-2) \varepsilon^{2}+2}{2} \bar{\xi}+\frac{(m-2) \varepsilon^{2}}{2} \mu\right)\right]}{\cosh \left[\frac{d^{\prime}}{\sigma}\left(\xi_{k}-\frac{(m-2) \varepsilon^{2}+2}{2} \bar{\xi}+\frac{(m-2) \varepsilon^{2}}{2} \mu\right)\right]}
$$

where $\bar{\xi}$ is the mean of all $m$ observations of one trial

$$
\bar{\xi}=\frac{1}{m} \sum_{j=1}^{m} \xi_{j} .
$$

If there is no bias, the response will be " $i$ " if and only if

$$
\mathcal{L}_{i, k}(\boldsymbol{\xi})>1
$$

is true for all $k$. This is identical to the following: Respond " $i$ " as the odd stimulus alternative if and only if $\cosh \left[\frac{d^{\prime}}{\sigma} Y_{i}\right]$ is equal to or greater than $\cosh \left[\frac{d^{\prime}}{\sigma} Y_{k}\right]$, where

$$
Y_{i}=\xi_{i}-\frac{(m-2) \varepsilon^{2}+2}{2} \bar{\xi}+\frac{(m-2) \varepsilon^{2}}{2} \mu .
$$

Because $\cosh \left[\frac{d^{\prime}}{\sigma} Y_{i}\right]$ is monotonic with $\left|Y_{i}\right|$,

$$
Z_{i}=\left|\xi_{i}-\frac{(m-2) \varepsilon^{2}+2}{2} \bar{\xi}+\frac{(m-2) \varepsilon^{2}}{2} \mu\right|
$$

is also the optimum decision statistic. Then, the decision rule becomes as follows: Respond " $i$ " as the odd stimulus alternative if and only if $Z_{i}$ is equal to or greater than $Z_{k}$ for all $k$.

When $\sigma_{R}^{2}=0$ (hence $\varepsilon^{2}=1$ and $\rho=0$ ), we have:

$$
\begin{aligned}
Z_{i} & =\left|\xi_{i}-\frac{m}{2} \bar{\xi}+\frac{m-2}{2} \mu\right| \\
& =\frac{1}{2}\left|2 \xi_{i}-\sum_{j=1}^{m} \xi_{j}+(m-2) \mu\right| .
\end{aligned}
$$

When $\sigma_{R}^{2} \gg \sigma^{2}\left(\varepsilon^{2} \approx 0\right.$ and $\left.\rho \approx 1\right)$, we have:

$$
Z_{i}=\left|\xi_{i}-\bar{\xi}\right| \text {. }
$$

For $m=3$, the last expression is, in fact, equivalent to the triangular decision rule, which is as follows. Consider three observations ordered from smallest to largest, call them $x_{1}^{\prime}, x_{2}^{\prime}$, and $x_{3}^{\prime}$. Because they are ordered, $x_{2}^{\prime}$ is between $x_{1}^{\prime}$ and $x_{3}^{\prime}$. Suppose $x_{2}^{\prime}$ is above the mean of all three, then $x_{2}^{\prime}$ is closer to $x_{3}^{\prime}$ and $x_{1}^{\prime}$ is the proper choice for the odd stimulus. If $x_{2}^{\prime}$ is below the mean, then $x_{2}^{\prime}$ is closer to $x_{1}^{\prime}$, and $x_{3}^{\prime}$ is the proper choice for the odd stimulus. The preceding is simply a restatement of what is called the triangular decision rule (Bradley, 1963). With Equation 17 it is also easy to generalize the decision rule for the case of $m$ stimuli (cf. Craven, 1992).

From this perspective, we see that there exists also another optimal decision rule, not previously mentioned in the literature-namely, one where observations are uncorrelated or independent (Equation 16). Equation 17 represents the optimal decision rule if the vectors are strongly correlated. The correlation among the elements of the observation vector is the only quantity that determines the form of the optimal decision rule used in oddity tasks.

In order to determine the shape of the psychometric function, we return to Equation 5 and solve it for $\mathcal{L}_{1, k}(\xi)>1$. Note that-without loss of generality--we now work with the likelihood ratio of the odd stimulus being in the first interval instead of the $i$ th, relative to the odd one being in the $k$ th interval. The two subspaces of the ( $m$-dimensional) decision space are (Appendix A)

$$
\begin{aligned}
& \left\{\begin{array}{l}
\xi_{1}>\xi_{k} \\
\left(\xi_{1}-\mu\right)+\left(\xi_{k}-\mu\right)>\frac{\varepsilon^{2}(m-2)+2}{m} \sum_{j=1}^{m}\left(\xi_{j}-\mu\right) \\
k=2,3, \ldots, m .
\end{array}\right. \\
& \left\{\begin{array}{l}
\xi_{1}<\xi_{k} \\
\left(\xi_{1}-\mu\right)+\left(\xi_{k}-\mu\right)<\frac{\varepsilon^{2}(m-2)+2}{m} \sum_{j=1}^{m}\left(\xi_{j}-\mu\right)
\end{array}\right.
\end{aligned}
$$

If $\sigma_{R}^{2}=0(\rho=0)$, there is no correlation between the observations, hence $\varepsilon^{2}=1$, and Equation 18 reduces to

$$
\begin{aligned}
& \xi_{1}>\xi_{k} \text { and }\left(\xi_{1}-\mu\right)+\left(\xi_{k}-\mu\right)>\sum_{j=1}^{m}\left(\xi_{j}-\mu\right) \\
& k=2,3, \ldots, m . \\
& \xi_{1}>\xi_{k} \text { and }\left(\xi_{1}-\mu\right)+\left(\xi_{k}-\mu\right)>\sum_{j=1}^{m}\left(\xi_{j}-\mu\right)
\end{aligned}
$$

Equation 19 shows that, in order to come to an optimal decision, one has to have knowledge of the mean of $\mu_{A}$ and $\mu_{B}, \mu$, or, in other words, needs a point of reference.

If the intervals are strongly correlated $\left(\sigma_{R}^{2} \rightarrow \infty\right.$, thus $\rho \rightarrow 1$ and $\varepsilon^{2} \rightarrow 0$ ), Equation 18 reduces to

$$
\begin{aligned}
& \xi_{1}>\xi_{k} \text { and } \xi_{1}+\xi_{k}>\frac{2}{m} \sum_{j=1}^{m} \xi_{j} \\
& \qquad k=2,3, \ldots, m . \\
& \xi_{1}>\xi_{k} \text { and } \xi_{1}+\xi_{k}>\frac{2}{m} \sum_{j=1}^{m} \xi_{j}
\end{aligned}
$$

Now, knowledge of the mean $\mu$ is not required, and observations are compared relative to one another. 
The set of decision rules next can be used to determine the shape of the psychometric function (probability of a correct response $P(c)$ as a function of the sensitivity $d^{\prime}$ ). Assuming unbiased responding and a balanced experimental design, $P(c)$ is found by integration of the probability density function $p d f_{A B B} \ldots B(\mathbf{x})$ over the appropriate two subspaces:

$$
P(c)=\int \cdot . \int_{\text {two } m \text {-dimensional subspaces }} . \int d \mathbf{x} p d f_{A B B \ldots B}(\mathbf{x}),(21)
$$

where the integration boundaries are determined by Equation 18 . Note that there always exist two subspacesnamely, one where the sequence $A B B \ldots B$ is correctly perceived, and one where it is mistaken for $B A A \ldots A$, but where the response ("the first interval contains the odd stimulus") is nevertheless correct.

Unfortunately, in general Equation 21 is difficult to solve analytically. Only for a number of special cases were we able to derive an analytic expression of the psychometric function. This is done in the following sections.

\section{THREE-INTERVAL ODDITY PARADIGM}

In most experiments, only three alternatives are used $(m=3)$. In that case, the decision rule is often described in terms of a triangle in which the three stimuli are located at the vertices of the triangle. The sides of the triangle represent the "distances" between the stimulus alternatives. Because two of the stimuli are the same, they will tend to be closer to each other than to the different or "odd" stimulus. Thus, it is argued that a good decision procedure is to select the vertex most distant from the other two. Bradley (1963) and Frijters (1979b) have presented an analysis of this task based on that decision rule. Frijters, Kooistra, and Vereijken (1980) have published tables on $d^{\prime}$ as a function of the percentage of correct responses for the three-alternative oddity task. Craven (1992) has recently presented similar tables for the $m$-alternative oddity problem $(3 \leq m \leq 32)$. As we will see later on, all expressions and tables are based on the assumption of highly correlated observations $\left(\rho \approx 1\right.$ or $\left.\varepsilon^{2} \approx 0\right)$. Next we will derive the expressions for $\varepsilon^{2}=0$ and $\varepsilon^{2}=1$ (corresponding to $\rho=1$ and $\rho=0$, respectively). We were not able to derive a general expression for $0<\varepsilon^{2}<1$.

\section{Uncorrelated Observations: $\varepsilon^{2}=1$}

If there is no rove, $\sigma_{R}^{2}=0(\rho=0)$ and thus $\varepsilon^{2}=1$. The observations are uncorrelated; thus the covariance matrix $\Sigma^{2}$ is a diagonal matrix. Insertion of $m=3$ into Equation 19 yields the decision rules to respond " 1 ," being

$$
\left\{\begin{array} { l } 
{ x _ { 1 } > x _ { 2 } } \\
{ x _ { 1 } > x _ { 3 } } \\
{ x _ { 2 } < \mu } \\
{ x _ { 3 } < \mu }
\end{array} \left\{\begin{array}{l}
x_{1}<x_{2} \\
x_{1}<x_{3} \\
x_{2}>\mu \\
x_{3}>\mu
\end{array} .\right.\right.
$$

The probability of a correct response, given by Equation 21 , can be determined with the constraints given in Equation 22 . Integrating over the two subspaces is not very difficult, but some insight is required to determine the inte- gration boundaries correctly. The psychometric function becomes

$$
\begin{aligned}
P\left(c, d^{\prime}\right)= & \int_{\mu}^{\infty} d x_{1} \int_{-\infty}^{\mu} d x_{2} \int_{-\infty}^{\mu} d x_{3} p d f_{A B B}(\mathbf{x}) \\
& +\int_{-\infty}^{\mu} d x_{1} \int_{-\infty}^{x_{1}} d x_{2} \int_{-\infty}^{x_{1}} d x_{3} p d f_{A B B}(\mathbf{x}) \\
& +\int_{-\infty}^{\mu} d x_{1} \int_{\mu}^{\infty} d x_{2} \int_{\mu}^{\infty} d x_{3} p d f_{A B B}(\mathbf{x}) \\
& +\int_{\mu}^{\infty} d x_{1} \int_{x_{1}}^{\infty} d x_{2} \int_{x_{1}}^{\infty} d x_{3} p d f_{A B B}(\mathbf{x})
\end{aligned}
$$

The expression for $p d f_{A B B}(\mathbf{x})$, given in Appendix A, is particularly simple in the present case of uncorrelated observations. Rearranging terms and using the definition of $d^{\prime}$ will yield the expression for the psychometric function

$$
\begin{aligned}
P\left(c, d^{\prime}\right)= & \Phi^{3}\left(\frac{d^{\prime}}{2}\right)+\int_{-\infty}^{-d^{\prime} / 2} \phi(u) \Phi^{2}\left(u+d^{\prime}\right) d u \\
& +\left[1-\Phi\left(\frac{d^{\prime}}{2}\right)\right]^{3}+\int_{-d^{\prime} / 2}^{\infty} \phi(u)\left[1-\Phi\left(u+d^{\prime}\right)\right]^{2} d u
\end{aligned}
$$

where $\phi(t)$ and $\Phi(t)$ are the Normal and the Cumulative Normal distributions, respectively:

$$
\begin{gathered}
\phi(t)=\frac{1}{\sqrt{2 \pi}} e^{-\frac{1}{2} t^{2}} \\
\Phi(t)=\int_{-\infty}^{t} \phi(u) d u .
\end{gathered}
$$

Equation 24 shows that the shape of the psychometric function does not depend on $\mu$. However, it is still necessary for the observer to know the reference value $\mu$ in order to be able to make an optimum decision - that is, to set the boundaries correctly.

\section{Highly Correlated Observations: $\varepsilon^{2} \approx 0$}

If the rove is large in comparison with the differences between two stimuli, $\sigma_{R}^{2}$ is very large in comparison with $\sigma^{2}$, such that $\varepsilon^{2} \approx 0(\rho \approx 1)$. Now we have to use the decision rules of Equation 20. Although we could make the same exercise as in the previous section, it is more convenient to first make a coordinate transformation such that the observations become uncorrelated (which is always possible because the covariance matrix is symmetric). This not only gives us more insight, but also makes it easier to derive an analytic expression of the psychometric function. The general expressions for the coordinate transformationwhich is in fact a rotation such that the $x_{1}$-axis is placed on the $x_{1}=x_{2}=\ldots=x_{m}$-axis-are given in Appendix B. Also given are the coordinates of the new expected values as well as the new decision boundaries. In the present case of $m=$ 3 and $\varepsilon^{2}=0$, the expected value $v$ for sequence $A B B$ is

$$
v_{A B B}=\left[\begin{array}{l}
\left(\mu_{A}-2 \mu_{B}\right) / \sqrt{3} \\
\left(\mu_{B}-\mu_{A}\right) / \sqrt{2} \\
\left(\mu_{B}-\mu_{A}\right) / \sqrt{6}
\end{array}\right],
$$

and the boundaries are 


$$
\left\{\begin{array} { l } 
{ z _ { 3 } < z _ { 2 } \sqrt { 3 } } \\
{ z _ { 3 } > 0 } \\
{ z _ { 2 } > 0 } \\
{ z _ { 3 } > - \frac { 1 } { \sqrt { 3 } } z _ { 2 } }
\end{array} \quad \left\{\begin{array}{l}
z_{3}>z_{2} \sqrt{3} \\
z_{3}<0 \\
z_{2}<0 \\
z_{3}<-\frac{1}{\sqrt{3}} z_{2}
\end{array} .\right.\right.
$$

The boundaries define two subspaces in the $\left(z_{2}, z_{3}\right)$ plane and are shown as shaded areas in Figure 1. As is shown in Appendix $\mathrm{B}, \sigma_{R}^{2}$ is situated in the $z_{1}$ direction only. Because $\sigma_{R}^{2}$ is very large, the density function in the $z_{1}$ direction is very broad and flat, so it becomes quite unimportant where on the $z_{1}$-axis the observation falls. Therefore, $z_{1}$ does not occur in the expressions for the decision boundaries. The psychometric function is now relatively easy to determine and is obtained by integration of the probability density function over the two shaded areas in Figure 1.

$$
\begin{gathered}
P\left(c, d^{\prime}\right)=\int_{-\infty}^{\infty} d z_{1} \int_{0}^{\infty} d z_{2} \int_{-\infty}^{z_{2} \sqrt{3}} d z_{3} p d f_{A B B}(\mathbf{z}) \\
+\int_{-\infty}^{\infty} d z_{1} \int_{-\infty}^{0} d z_{2} \int_{-z_{2} \sqrt{3}}^{0} d z_{3} p d f_{A B B}(\mathbf{z}) \\
=\int_{-d^{\prime} / \sqrt{2}}^{\infty} d u \phi(u)\left[\Phi\left(\frac{d^{\prime}}{\sqrt{6}}\right)-\Phi\left(-u \sqrt{3}-d^{\prime} \sqrt{\frac{2}{3}}\right)\right] \\
+\int_{-\infty}^{-d^{\prime} / \sqrt{2}} d u \phi(u)\left[\Phi\left(-u \sqrt{3}-d^{\prime} \sqrt{\frac{2}{3}}\right)-\Phi\left(\frac{d^{\prime}}{\sqrt{6}}\right)\right] .
\end{gathered}
$$

It can be shown that Equation 30 is identical to the one obtained by Bradley (1963) and Frijters (1979b).

\section{TABLES FOR THE $M$-INTERVAL ODDITY PARADIGM}

Craven (1992) has published a table relating $d^{\prime}$ and the probability of being correct in the general $m$-interval odd-

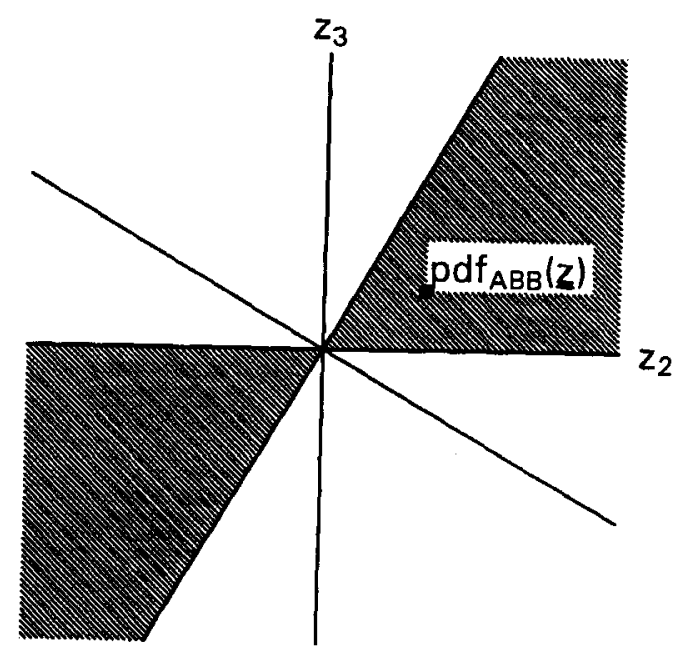

Figure 1. Decision space for the oddity paradigm with fully correlated observations $\left(\varepsilon^{2}=0\right)$. In order to obtain the probability on a correct response $P(c)$, the probability density function $p d f_{A B B}(z)$ has to be integrated over the shaded areas in the $\left(z_{2}, z_{3}\right)$ plane. ity task. Also, Hacker and Ratcliff (1979) have tabulated $d^{\prime}$ as a function of $P(c)$ for the $m$ AFC paradigm. The only psychometric function that needs to be tabulated is the one with $\varepsilon^{2}=1$. We simulated the decision rules by using Monte Carlo procedures for the $m \mathrm{AFC}$ task and the $m$-interval oddity task for both $\varepsilon^{2}=0$ and $\varepsilon^{2}=1(m=3,4,5)$. The number of trials used in the simulations was $1,000,000$ for each value of $d^{\prime}$. Here--in contrast to previous publications--the obtained probability of a correct response, given in Table 1, is tabulated as a function of $d^{\prime}$.

The data in Table 1 show good agreement with those of Craven (1992) and Hacker and Ratcliff (1979). ${ }^{2}$ The psychometric functions are plotted in Figure 2. It can be seen that a three-interval oddity task is much more difficult than a 3AFC task, as Frijters (1979a) pointed out. Furthermore, knowledge of the stimuli- that is, knowing $\mu$--indeed increases the probability of a correct response. As $m$ increases, the psychometric function for the $\varepsilon^{2}=1$ oddity task shifts from the $\varepsilon^{2}=0$ function toward the $m \mathrm{AFC}$ function.

\section{$P(c)$ AS A FUNCTION OF $\rho$}

It is of interest to know in what way the probability of a correct response is affected by $\sigma_{R}^{2}$, the size of the common variability. Therefore, $P(c)$ was calculated through simulation, for $m=3,4,5$, as a function of $\rho$. As an example, we took $d^{\prime}=3$. The results are plotted in Figure 3 . The shape of the curves in Figure 3 is similar for other values of $d^{\prime}$.

It can be seen from Figure 3 that a variation in the order of magnitude of the difference between the two stimuli $\left(\sigma_{R} / \sigma=1\right.$ or $\left.\rho=0.5\right)$ already produces a considerable shift in $P(c)$, generating a psychometric function that is halfway between that of the highly correlated and the uncorrelated function. The just noticeable difference for frequency changes is about $0.1 \%-1 \%$ (for frequencies beyond $500 \mathrm{~Hz}$; Wier, Jesteadt, \& Green, 1977). In our example of frequency discrimination, this indicates that roving the standard frequency by about $0.1 \%-1 \%$ should cause noticeable deterioration in performance.

\section{HOW CAN WE DETERMINE THE DECISION RULE USED IN A TYPICAL ODDITY TASK?}

In any oddity task, it would be interesting to know what decision rule is actually used by the observer. Is the decision rule the optimum one for uncorrelated observations $\left(\varepsilon^{2}=1\right)$, or does the observer use the triangular rule expressed by Frijters $(1979 b)\left(\varepsilon^{2}=0\right)$ ? A technique recently suggested by Berg (1989), called the conditional on a single stimulus (COSS), may be useful in trying to answer these questions. We have not attempted to use the technique experimentally, but computer simulations of these two different decision rules suggest that it may be of value. Human observers, of course, may follow rules that lie somewhere between the two decision rules studied in this paper. Our presentation will explain the application of a correlation technique suggested by Richards and Zhu (1994) and by 
Table 1

$P(c)$ as a Function of $d^{\prime}$ for the $m$ AFC Paradigm $(m=3,4,5)$, and the Oddity Paradigm ( $m=3,4,5$ and $\varepsilon^{2}$ Is Either 0 or 1 )

\begin{tabular}{|c|c|c|c|c|c|c|c|c|c|}
\hline \multirow[b]{3}{*}{$d^{\prime}$} & \multicolumn{3}{|c|}{$m=3$} & \multicolumn{3}{|c|}{$m=4$} & \multicolumn{3}{|c|}{$m=5$} \\
\hline & \multirow[b]{2}{*}{$\mathrm{AFC}$} & \multicolumn{2}{|c|}{ Oddity } & \multirow[b]{2}{*}{$\mathrm{AFC}$} & \multicolumn{2}{|c|}{ Oddity } & \multirow[b]{2}{*}{$\mathrm{AFC}$} & \multicolumn{2}{|c|}{ Oddity } \\
\hline & & $\overline{\varepsilon^{2}}=0$ & $\varepsilon^{2}=1$ & & $\varepsilon^{2}=0$ & $\overline{\varepsilon^{2}=1}$ & & $\varepsilon^{2}=0$ & $\varepsilon^{2}=1$ \\
\hline 0.0 & 0.333 & 0.333 & 0.333 & 0.250 & 0.250 & 0.250 & 0.200 & 0.200 & 0.200 \\
\hline 0.1 & 0.362 & 0.334 & 0.335 & 0.277 & 0.252 & 0.252 & 0.224 & 0.201 & 0.202 \\
\hline 0.2 & 0.391 & 0.337 & 0.338 & 0.304 & 0.255 & 0.257 & 0.249 & 0.205 & 0.208 \\
\hline 0.3 & 0.422 & 0.342 & 0.345 & 0.333 & 0.261 & 0.267 & 0.278 & 0.211 & 0.219 \\
\hline 0.4 & 0.452 & 0.348 & 0.353 & 0.362 & 0.268 & 0.279 & 0.305 & 0.221 & 0.234 \\
\hline 0.5 & 0.483 & 0.356 & 0.364 & 0.393 & 0.279 & 0.295 & 0.334 & 0.232 & 0.252 \\
\hline 0.6 & 0.512 & 0.365 & 0.376 & 0.424 & 0.292 & 0.313 & 0.366 & 0.246 & 0.275 \\
\hline 0.7 & 0.543 & 0.376 & 0.392 & 0.456 & 0.306 & 0.334 & 0.397 & 0.261 & 0.299 \\
\hline 0.8 & 0.574 & 0.389 & 0.409 & 0.487 & 0.321 & 0.358 & 0.428 & 0.279 & 0.327 \\
\hline 0.9 & 0.604 & 0.403 & 0.427 & 0.520 & 0.339 & 0.385 & 0.461 & 0.299 & 0.357 \\
\hline 1.0 & 0.633 & 0.418 & 0.446 & 0.552 & 0.360 & 0.413 & 0.495 & 0.321 & 0.391 \\
\hline 1.1 & 0.663 & 0.434 & 0.468 & 0.583 & 0.381 & 0.443 & 0.528 & 0.344 & 0.426 \\
\hline 1.2 & 0.690 & 0.452 & 0.491 & 0.615 & 0.404 & 0.474 & 0.559 & 0.368 & 0.460 \\
\hline 1.3 & 0.716 & 0.468 & 0.513 & 0.644 & 0.426 & 0.506 & 0.591 & 0.394 & 0.496 \\
\hline 1.4 & 0.741 & 0.488 & 0.536 & 0.673 & 0.449 & 0.538 & 0.624 & 0.420 & 0.533 \\
\hline 1.5 & 0.765 & 0.507 & 0.560 & 0.702 & 0.475 & 0.571 & 0.653 & 0.447 & 0.568 \\
\hline 1.6 & 0.788 & 0.525 & 0.584 & 0.729 & 0.499 & 0.603 & 0.683 & 0.475 & 0.604 \\
\hline 1.7 & 0.810 & 0.546 & 0.608 & 0.755 & 0.524 & 0.635 & 0.711 & 0.503 & 0.639 \\
\hline 1.8 & 0.831 & 0.565 & 0.631 & 0.779 & 0.549 & 0.665 & 0.738 & 0.531 & 0.673 \\
\hline 1.9 & 0.848 & 0.585 & 0.654 & 0.802 & 0.575 & 0.696 & 0.764 & 0.559 & 0.705 \\
\hline 2.0 & 0.865 & 0.605 & 0.677 & 0.823 & 0.600 & 0.725 & 0.788 & 0.586 & 0.735 \\
\hline 2.1 & 0.881 & 0.624 & 0.699 & 0.842 & 0.624 & 0.751 & 0.810 & 0.614 & 0.764 \\
\hline 2.2 & 0.896 & 0.645 & 0.722 & 0.860 & 0.649 & 0.777 & 0.831 & 0.640 & 0.791 \\
\hline 2.3 & 0.909 & 0.663 & 0.742 & 0.877 & 0.672 & 0.801 & 0.851 & 0.667 & 0.816 \\
\hline 2.4 & 0.921 & 0.682 & 0.763 & 0.893 & 0.695 & 0.824 & 0.869 & 0.692 & 0.839 \\
\hline 2.5 & 0.931 & 0.700 & 0.781 & 0.907 & 0.716 & 0.845 & 0.885 & 0.716 & 0.859 \\
\hline 2.6 & 0.941 & 0.718 & 0.799 & 0.919 & 0.738 & 0.864 & 0.900 & 0.740 & 0.878 \\
\hline 2.7 & 0.949 & 0.734 & 0.816 & 0.930 & 0.758 & 0.882 & 0.914 & 0.762 & 0.895 \\
\hline 2.8 & 0.957 & $0.75 !$ & 0.832 & 0.941 & 0.778 & 0.898 & 0.925 & 0.783 & 0.910 \\
\hline 2.9 & 0.963 & 0.766 & 0.847 & 0.949 & 0.796 & 0.911 & 0.936 & 0.803 & 0.924 \\
\hline 3.0 & 0.968 & 0.783 & 0.862 & 0.957 & 0.813 & 0.924 & 0.945 & 0.821 & 0.935 \\
\hline 3.1 & 0.974 & 0.795 & 0.874 & 0.963 & 0.829 & 0.935 & 0.953 & 0.839 & 0.945 \\
\hline 3.2 & 0.978 & 0.810 & 0.886 & 0.969 & 0.845 & 0.945 & 0.961 & 0.855 & 0.954 \\
\hline 3.3 & 0.981 & 0.823 & 0.897 & 0.974 & 0.859 & 0.953 & 0.967 & 0.870 & 0.962 \\
\hline 3.4 & 0.985 & 0.836 & 0.908 & 0.978 & 0.873 & 0.961 & 0.972 & 0.884 & 0.969 \\
\hline 3.5 & 0.987 & 0.848 & 0.917 & 0.982 & 0.885 & 0.967 & 0.977 & 0.897 & 0.974 \\
\hline 3.6 & 0.990 & 0.858 & 0.926 & 0.985 & 0.896 & 0.973 & 0.981 & 0.908 & 0.979 \\
\hline 3.7 & 0.991 & 0.870 & 0.934 & 0.988 & 0.907 & 0.978 & 0.984 & 0.919 & 0.983 \\
\hline 3.8 & 0.993 & 0.879 & 0.941 & 0.990 & 0.916 & 0.982 & 0.987 & 0.928 & 0.985 \\
\hline 3.9 & 0.995 & 0.889 & 0.947 & 0.992 & 0.925 & 0.985 & 0.990 & 0.938 & 0.989 \\
\hline 4.0 & 0.995 & 0.898 & 0.953 & 0.993 & 0.933 & 0.988 & 0.992 & 0.945 & 0.991 \\
\hline 4.1 & 0.996 & 0.906 & 0.959 & 0.995 & 0.941 & 0.990 & 0.993 & 0.952 & 0.993 \\
\hline 4.2 & 0.997 & 0.914 & 0.964 & 0.996 & 0.948 & 0.992 & 0.995 & 0.958 & 0.994 \\
\hline 4.3 & 0.998 & 0.921 & 0.968 & 0.997 & 0.953 & 0.994 & 0.996 & 0.964 & 0.995 \\
\hline 4.4 & 0.998 & 0.927 & 0.972 & 0.997 & 0.959 & 0.995 & 0.996 & 0.969 & 0.996 \\
\hline 4.5 & 0.999 & 0.934 & 0.976 & 0.998 & 0.964 & 0.996 & 0.997 & 0.973 & 0.997 \\
\hline 4.6 & 0.999 & 0.939 & 0.978 & 0.998 & 0.968 & 0.997 & 0.998 & 0.977 & 0.998 \\
\hline 4.7 & 0.999 & 0.946 & 0.981 & 0.999 & 0.972 & 0.997 & 0.998 & 0.980 & 0.998 \\
\hline 4.8 & 0.999 & 0.950 & 0.983 & 0.999 & 0.976 & 0.998 & 0.999 & 0.983 & 0.999 \\
\hline 4.9 & 0.999 & 0.955 & 0.986 & 0.999 & 0.979 & 0.998 & 0.999 & 0.986 & 0.999 \\
\hline 5.0 & 1.000 & 0.959 & 0.987 & 0.999 & 0.981 & 0.999 & 0.999 & 0.988 & 0.999 \\
\hline
\end{tabular}

Lutfi (1995), but we will refer to it as the COSS procedure. For a more detailed discussion of these techniques and their limitations, the original papers should be consulted.

In these techniques, each of the stimulus values presented in the oddity task is perturbed or altered by an independent random variable. The perturbation variable is Gaussian with zero mean. For example, suppose we have a three-interval frequency discrimination oddity task; then two tones would have the same frequency, and one would have a different frequency. Once the perturbation is applied, all tones will have different frequencies. This need not be a problem because the standard deviation of the perturbation is chosen 

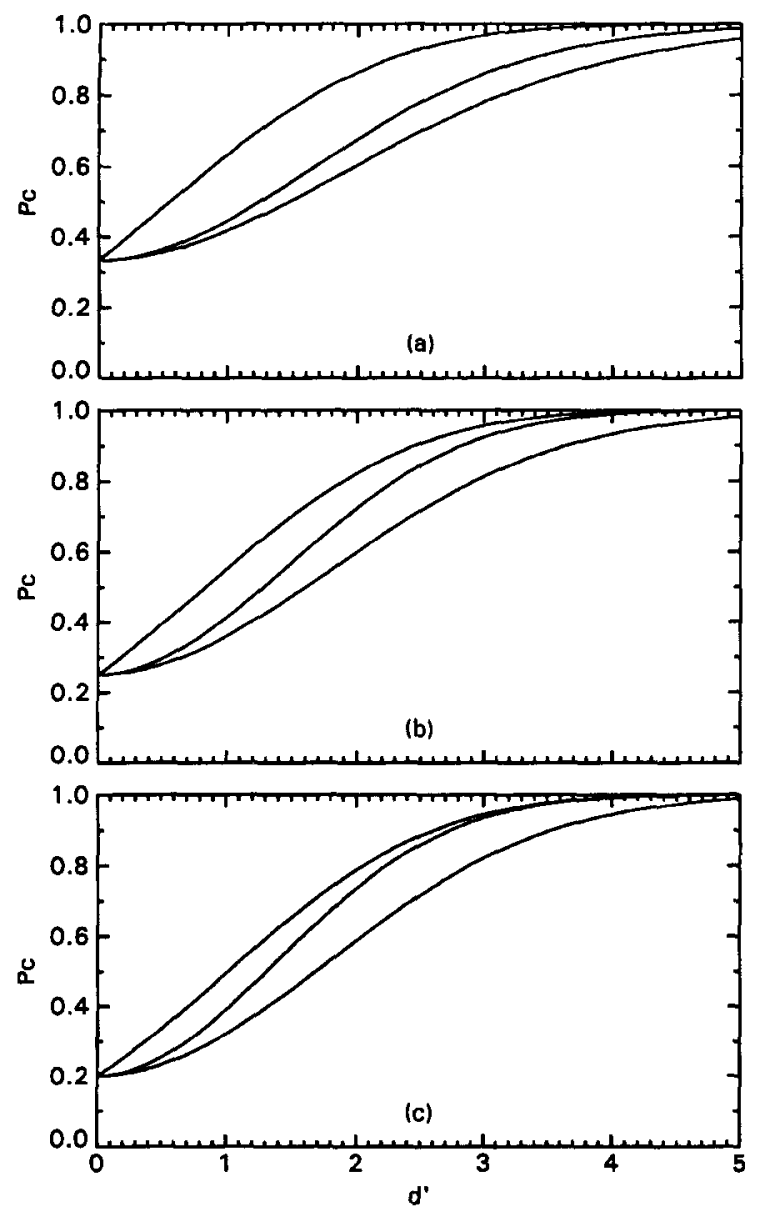

Figure 2. Psychometric functions for the $m$ AFC paradigm (upper curves), oddity paradigm with $\varepsilon^{2}=1$ (middle curves), and the oddity paradigm with $\varepsilon^{2}=0$ (lower curves). (a) 3-interval, (b) 4-interval, (c) 5-interval paradigm.

to be small. In other applications, we have found that the perturbations were barely noticed by the observer (Berg \& Green, 1990).

In the COSS analysis, the observer's response on each trial is correlated with the different perturbation values. In the case of the oddity task, it is convenient to code the response as either correct or incorrect (e.g., $R=1$ for a correct response and $R=0$ for an incorrect response). At the conclusion of a set of trials we can compute three correlations. One is between the response and the perturbation for the odd stimulus, and the second and third correlations are computed between the response and the perturbations on the remaining (non-odd) stimuli. Because there is nothing to distinguish between the two non-odd stimuli, we average the two correlations in what follows.

Figure 4 shows computer simulations using the two decision rules: Equation 30 for the correlated (triangular) rule and Equation 24 for the uncorrelated decision rule. The standard deviation of the stimulus perturbation, $\sigma_{p}$, was chosen at 1.5 times the threshold for the signal (measured without perturbation) in this task-that is, $\sigma_{p}=1.5 \sigma$. The percentage of correct responses should be about $76 \%$ for the optimum decision rule and $69 \%$ for the triangular rule. Each point is the correlation, computed over a set of 200 trials, between the binary variable $R$, coding whether the response was correct or incorrect, and the perturbation of the stimulus $\sigma_{p}$. The results obtained with the triangular rule are shown as circles ("O"), and the results obtained with the other rule are shown as "+." For example, with the triangular rule, correlation between the response variable and the perturbation of the non-odd stimuli is about -0.15 , on the average. The correlation associated with the odd stimulus is on the average about 0.3 . When the other decision rule is employed, these two correlations are on the average -0.35 and 0.1 .

The cluster of points is based on 2,000 simulations, each employing 200 trials. The line is a simple linear function $(y=-0.6 x+0.05)$ that was adjusted empirically to separate the two clusters. The two error percentages are shown in Figure 4; that is, $2.2 \%$ and $0.6 \%$ of the points fall on the wrong side of the line. If the correlations are computed over a greater number of trials, the ability to discriminate between the two decision rules is even better. With 300 responses instead of 200 , the misclassification rate was less than $1 \%$, and it was virtually impossible to measure the misclassification rate when 1,000 trials were used. When this technique is used with human subjects, some care must be taken to adjust the parameters of the task so that useful correlations will result. One important quantity is the percentage of correct responses made in the course of the experiment. Obviously, if the percentage correct value is very high (near $100 \%$ ), the responses produced

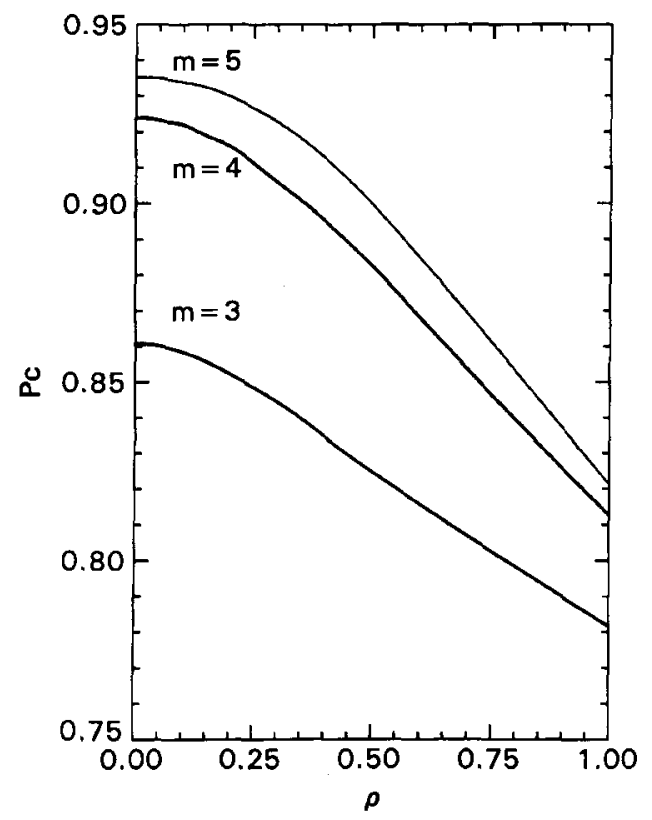

Figure 3. Probability of a correct response $P(c)$ as a function of $\rho$, the correlation between the observations. $m=3,4,5$. 


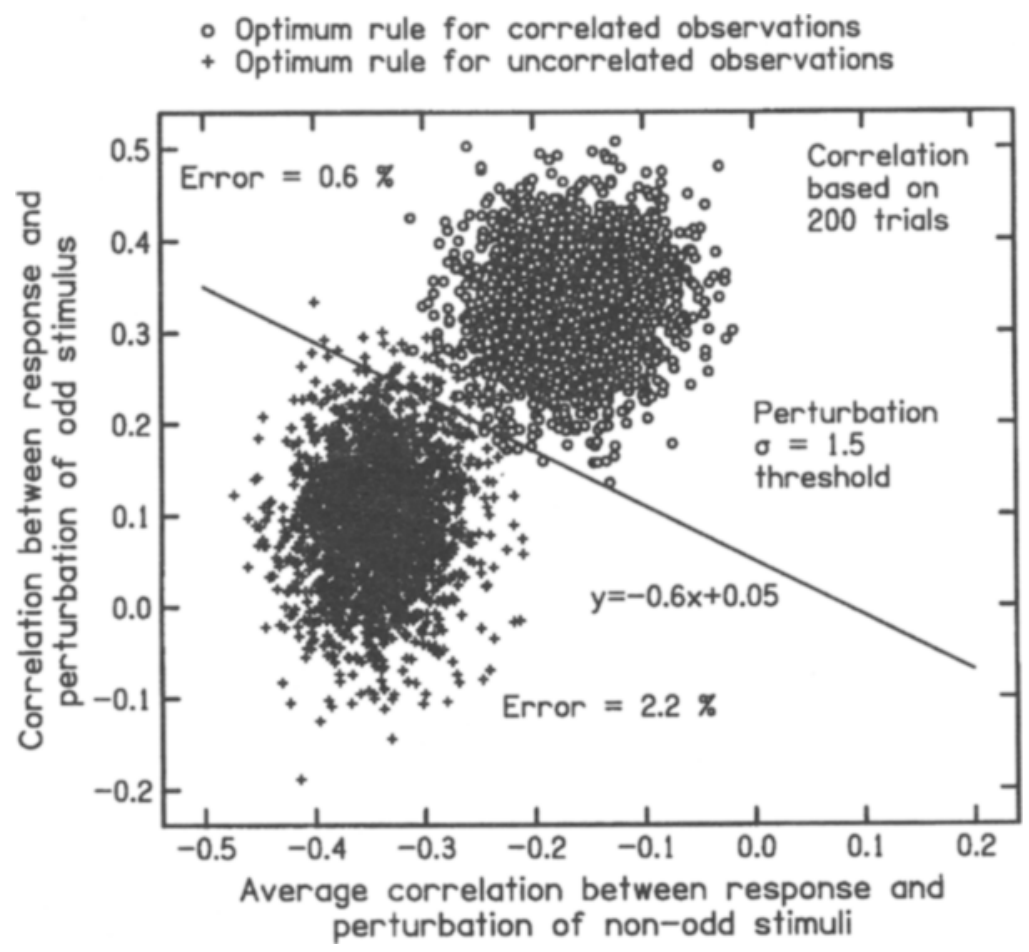

Figure 4. Correlation between response and perturbation of the odd stimulus as a function of the average correlation between response and perturbation of nonodd stimuli. "+" denotes results obtained with the $\varepsilon^{2}=1$ rule; "O" denotes results obtained with the $\varepsilon^{2}=0$ rule.

by the two decision rules will be virtually the same, and there will be no differences between the correlations obtained with the different decision rules. The percentage of correct responses is a joint function of the stimulus difference and the standard deviation used in the perturbation. Our recommendation is to use a perturbation that is about 1.5 times larger than the threshold value $\left(d^{\prime}=1\right)$ for the signal without any perturbations. We do not recommend large values of perturbation, because the observer might doubt the validity of the feedback and employ idiosyncratic response patterns. In the simulation, the percentage of correct responses was about $70 \%-80 \%$, and it was easy to distinguish between the different decision rules.

\section{SUMMARY}

In this paper, we have derived a general decision rule for the $m$-interval oddity task. In particular, analytic expressions were derived in case $m=3$ for the highly correlated and the uncorrelated cases. It has been shown that the oddity task will be performed better if the observer is acquainted with the stimuli, so that the intervals can be perceived independently. Furthermore, tables have been presented for the oddity task in cases where $m=3,4$, and 5 . Lastly, a method has been proposed that may be adopted in order to determine which decision rule is used by an observer.

\section{REFERENCES}

BERG, B. G. (1989). Analysis of weights in multiple observation tasks. Journal of the Acoustical Society of America, 86, 1743-1746.

BerG, B. G., \& GreEN, D. M. (1990). Spectral weights in profile listening. Journal of the Acoustical Society of America, 88, 758-766.

BRADLEY, R. A. (1963). Some relationships among sensory difference tests. Biometrics, 19, 385-397.

Craven, B. J. (1992). A table of $d^{\prime}$ for $M$-alternative odd-man-out forced-choice procedures. Perception \& Psychophysics, \$1, 379-385.

Dai, H., Versfeld, N. J., \& GreEn, D. M. (1996). The optimum decision rules in the same-different paradigm. Perception \& Psychophysics, $\mathbf{5 8}, 1-9$.

Durlach, N. I., Braida, L. D., \& ITO, Y. (1986). Towards a model for discrimination of broadband signals. Journal of the Acoustical Society of America, 80, 63-72.

FRIJTERS, J. E. R. (1979a). The paradox of discriminatory nondiscriminators resolved. Chemical Senses \& Flavour, 4, 355-358.

FRIJTERS, J. E. R. (1979b). Variations of the triangular method and the relationship of its unidimensional probabilistic models to three-altemative forced-choice signal detection theory models. British Journal of Mathematical \& Statistical Psychology, 32, 229-241.

Frijters, J. E. R., Kooistra, A., \& Verejuken, P. F. G. (1980). Tables of $d^{\prime}$ for the triangular method and the 3-AFC signal detection procedure. Perception \& Psychophysics, 27, 176-178.

GREEN, D. M., \& SWETS, J. A. (1988). Signal detection theory and psychophysics. Los Altos, CA: Peninsula Publishing. 
HACKER, M. J., \& RATCLIFF, R. (1979). A revised table of $d^{\prime}$ for $M$ aiternative forced choice. Perception \& Psychophysics, 26, 168-170.

Lutfi, R. A. (1995). Correlation coefficients and correlation ratios as estimates of observer weights in multiple-observation tasks. Journal of the Acoustical Society of America, 97, 1333-1334.

Macmillan, N. A., \& Creelman, C. D. (1991). Detection theory: A user's guide. Cambridge: Cambridge University Press.

RICHARDS, V. M., \& ZHU, S. (1994). Relative estimates of combination weights, decision criteria, and internal noise based on correlation coefficients. Journal of the Acoustical Society of America, 95, 423434.

Wier, C. C., Jesteadt, W., \& Green, D. M. (1977). Frequency discrimination as a function of frequency and sensation level. Journal of the Acoustical Society of America, 61, 178-184.

\section{NOTES}

1. To illustrate the difference between the two tasks, the likelihood ratio for the $M A F C$ paradigm is

$$
\mathcal{L}_{i, k}^{m A F C}(\xi)=\frac{p d f_{A \ldots A B_{i} A \ldots A}(\xi)}{p d f_{A \ldots A B_{k} A \ldots A}(\boldsymbol{\xi})}
$$

2. We managed to derive an analytic expression for the psychometric function for $m=4$ and $\varepsilon^{2}=0$. In that case, the decision space is threedimensional and the psychometric function is given by

$$
P\left(c, d^{\prime}\right)=\Phi^{3}\left(\frac{d^{\prime}}{2}\right)+\left[1-\Phi\left(\frac{d^{\prime}}{2}\right)\right]^{3}
$$

\section{APPENDIX A}

\section{Derivation of the Likelihood Ratios}

The likelihood ratio of $\xi$ coming from a sequence with the odd stimulus in the $i$ th interval relative to one with the odd stimulus in the $k$ th interval is given by

$$
\mathcal{L}_{i, k}(\boldsymbol{\xi})=\frac{p d f_{B \ldots B A_{i} B \ldots B B}(\boldsymbol{\xi})+p d f_{A \ldots A B_{i} A \ldots A A}(\boldsymbol{\xi})}{p d f_{B \ldots \ldots A_{k} B \ldots B}(\boldsymbol{\xi})+p d f_{A \ldots A_{B_{k}} A . A}(\boldsymbol{\xi})} .
$$

If we directly omit all terms that are identical in all four density functions, we get

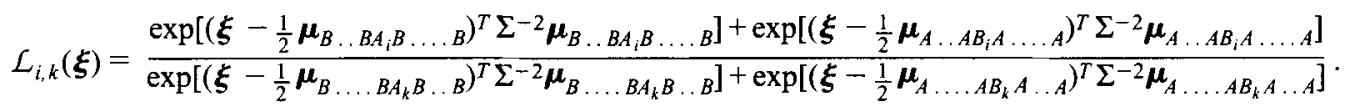

Here, $\mu_{B} \ldots B A_{i} B \ldots B$ is the vector $\left[\mu_{B} \ldots \mu_{B} \mu_{A} \mu_{B} \ldots \mu_{B}\right]^{T}\left(T\right.$ stands for transpose), with $\mu_{A}$ on the $i$ th position, and $\mu_{A}=E[\xi \mid A]$ and $\mu_{B}=E[\xi \mid B]$ are the expected values for $\xi$ given Stimulus $A$ and Stimulus $B$, respectively. Thus $\mu_{B \ldots B A_{i} B \ldots B}=E\left[\left.\xi\right|_{B \ldots B A_{i} B \ldots B}\right]$. The inverse matrix of $\Sigma^{2}$ is given by

$$
\Sigma^{-2}=\frac{1}{\sigma^{2}}\left[\begin{array}{cccccc}
1+b & b & \cdot & \cdot & \cdot & b \\
b & 1+b & & & \cdot \\
\cdot & & \cdot & & & \cdot \\
\cdot & & & \cdot & & \cdot \\
\cdot & & & & 1+b & b \\
b & . & . & . & b & 1+b
\end{array}\right],
$$

where

$$
b=-\frac{\sigma_{R}^{2}}{\sigma^{2}+m \sigma_{R}^{2}}=\frac{\varepsilon^{2}-1}{m},
$$

and $\varepsilon^{2}$ is defined as

$$
\varepsilon^{2}=\frac{\sigma^{2}}{\sigma^{2}+m \sigma_{R}^{2}}
$$

(The notation $\Sigma^{2}$ has been chosen since $\Sigma^{2}=\sigma^{2}$ for $m=1$. Also, we have used $\varepsilon^{2}$, rather than $\varepsilon$, merely to indicate that its value is always positive.) The relation between the correlation $\rho$ and $\sigma_{R}^{2}$ and $\varepsilon^{2}$ is

$$
\rho=\frac{\sigma_{R}^{2}}{\sigma^{2}+\sigma_{R}^{2}}=\frac{1-\varepsilon^{2}}{1+(m-1) \varepsilon^{2}} .
$$

Now it is not so very difficult to write out the individual terms in the exponent:

$$
\begin{aligned}
& -\frac{1}{2} \boldsymbol{\mu}_{B \ldots B A B \ldots B}^{T} \Sigma^{-2} \boldsymbol{\mu}_{B} \ldots B A B \ldots B=\frac{1}{2 m \sigma^{2}}\left\{\varepsilon^{2}\left[\mu_{A}+(m-1) \mu_{B}\right]^{2}+(m-1)\left(\mu_{B}-\mu_{A}\right)^{2}\right\} \\
& =-\frac{1}{2 m}\left\{\frac{\varepsilon^{2}}{4 \sigma^{2}}\left[(m-2) d^{\prime} \sigma+2 m \mu\right]^{2}+(m-1)\left(d^{\prime}\right)^{2}\right\},
\end{aligned}
$$


where $\mu$ and $d^{\prime}$ are defined as

$$
\mu=\frac{\mu_{A}+\mu_{B}}{2},
$$

and

$$
d^{\prime}=\frac{\mu_{B}-\mu_{A}}{\sigma} .
$$

From Equations $\mathrm{A} 9$ and $\mathrm{A} 10$ it can be deduced that $\mu_{A}$ and $\mu_{B}$ can be written as

$$
\left\{\begin{array}{l}
\mu_{A}=\mu-\frac{1}{2} d^{\prime} \sigma \\
\mu_{B}=\mu+\frac{1}{2} d^{\prime} \sigma
\end{array}\right.
$$

Also

$$
\begin{aligned}
\boldsymbol{\xi}_{B \ldots B A B \ldots B}^{T} \Sigma^{-2} \boldsymbol{\mu}_{B \ldots B A B \ldots B} & =\frac{1}{m \sigma^{2}}\left\{-m\left(\mu_{B}-\mu_{A}\right) \xi_{k}+\sum_{j=1}^{m}\left[\varepsilon^{2}\left(\mu_{A}+(m-1) \mu_{B}\right)+\left(\mu_{B}-\mu_{A}\right)\right] \xi_{j}\right\} \\
& =-\frac{\xi_{k} d^{\prime}}{\sigma}+\sum_{j=1}^{m} \frac{\xi_{j}}{2 m \sigma^{2}}\left\{\left(2+(m-2) \varepsilon^{2}\right) d^{\prime} \sigma+2 m \varepsilon^{2} \mu\right\} .
\end{aligned}
$$

The same can be calculated for a sequence with $A \ldots A B A \ldots A$, which gives

$$
\begin{gathered}
-\frac{1}{2} \boldsymbol{\mu}_{A \ldots A B A \ldots A}^{T} \Sigma^{-2} \boldsymbol{\mu}_{A \ldots A B A \ldots A}=-\frac{1}{2 m}\left\{\frac{\varepsilon^{2}}{4 \sigma^{2}}\left[(2-m) d^{\prime} \sigma+2 m \mu\right]^{2}+(m-1)\left(d^{\prime}\right)^{2}\right\} \\
\boldsymbol{\xi}_{A \ldots A B A \ldots A}^{T} \Sigma^{-2} \boldsymbol{\mu}_{A \ldots A B A \ldots A}=\frac{\xi_{k} d^{\prime}}{\sigma}-\sum_{j=1}^{m} \frac{\xi_{j}}{2 m \sigma^{2}}\left\{\left(2+(m-2) \varepsilon^{2}\right) d^{\prime} \sigma+2 m \mu\right\} .
\end{gathered}
$$

Insertion of Equations $\mathrm{A} 8, \mathrm{~A} 13, \mathrm{~A} 14$, and $\mathrm{A} 15$ into Equation $\mathrm{A} 2$ yields - after quite a lot of rearranging and omission of terms that are identical in all four exponents

$$
\mathcal{L}_{i, k}(\boldsymbol{\xi})=\frac{\cosh \left[\frac{d^{\prime}}{2 m \sigma} \sum_{j=1}^{m}\left\{2\left(\xi_{j}-\xi_{i}\right)+(m-2) \varepsilon^{2}\left(\xi_{j}-\mu\right)\right\}\right]}{\cosh \left[\frac{d^{\prime}}{2 m \sigma} \sum_{j=1}^{m}\left\{2\left(\xi_{j}-\xi_{k}\right)+(m-2) \varepsilon^{2}\left(\xi_{j}-\mu\right)\right\}\right]},
$$

which is Equation 5 in the main text. The hyperbolic-cosine function is defined as

$$
\cosh (z)=\frac{e^{-z}+e^{z}}{2}
$$

Solving Equation A16 for $\mathcal{L}_{i, k}(\boldsymbol{\xi})>1$ is straightforward, if one bears in mind that $\operatorname{solving} \cosh \left(p_{i}\right)>\cosh \left(p_{k}\right)$ is equivalent to solving $p_{i}^{2}>p_{k}^{2}$, and that

$$
\left[\sum_{j=1}^{m} p_{j}\right]^{2}=\sum_{j=1}^{m} \sum_{j^{\prime}=1}^{m} p_{j} p_{j^{\prime}}
$$

Departing from Equation A16 we thus get

$$
\left[\sum_{j=1}^{m} \xi_{j}-\xi_{i}+c_{j}\right]^{2}>\left[\sum_{j=1}^{m} \xi_{j}-\xi_{k}+c_{j}\right]^{2}
$$

where

$$
c_{j}=\frac{1}{2}(m-2) \varepsilon^{2}\left(\xi_{j}-\mu\right) .
$$

Elaborating the squared terms yields, with the use of Equation $\mathrm{A} 18$,

$$
\sum_{j=1}^{m} \sum_{j^{\prime}=1}^{m}\left(\xi_{j}-\xi_{i}+c_{j}\right)\left(\xi_{j^{\prime}}-\xi_{i}+c_{j^{\prime}}\right)>\sum_{j=1}^{m} \sum_{j^{\prime}=1}^{m}\left(\xi_{j}-\xi_{k}+c_{j}\right)\left(\xi_{j^{\prime}}-\xi_{k}+c_{j^{\prime}}\right) .
$$

Rearranging terms yields 


$$
m^{2}\left(\xi_{k}-\xi_{i}\right)\left[\left(\xi_{i}+\xi_{k}\right)+\frac{2}{m} \sum_{j=1}^{m} \xi_{j}+c_{j}\right]>0
$$

In order to satisfy Equation A22, the two terms both have to be either positive or negative, which gives us two subspaces. Insertion of the definition of $c_{j}$, as well as some rearranging, finally gives us

$$
\begin{aligned}
& \left\{\begin{array}{l}
\xi_{i}>\xi_{k} \\
\left(\xi_{i}-\mu\right)+\left(\xi_{k}-\mu\right)>\frac{\varepsilon^{2}(m-2)+2}{m} \sum_{j=1}^{m}\left(\xi_{j}-\mu\right)
\end{array},\right. \\
& \left\{\begin{array}{l}
\xi_{i}<\xi_{k} \\
\left(\xi_{i}-\mu\right)+\left(\xi_{k}-\mu\right)<\frac{\varepsilon^{2}(m-2)+2}{m} \sum_{j=1}^{m}\left(\xi_{j}-\mu\right)
\end{array}\right.
\end{aligned}
$$

which is, for $i=1$, identical to Equation 18 in the main text.

As an example, we write out the probability density function for $m=3$ :

$$
p d f_{A B B}(\mathbf{x})=\frac{1}{\sqrt{(2 \pi)^{m}\left|\Sigma^{2}\right|}} \exp \left[-\frac{1}{2}\left(\mathbf{x}-\boldsymbol{\mu}_{A B B}\right)^{r} \Sigma^{-2}\left(\mathbf{x}-\boldsymbol{\mu}_{A B B}\right)\right] .
$$

The determinant of $\Sigma^{2}$ is simply $\left|\Sigma^{2}\right|=\sigma^{2 m} / \varepsilon^{2}$, which can most easily be verified by inspection of Equation B3 of Appendix B. Thus for $m=3,\left|\Sigma^{2}\right|=\sigma^{6} / \varepsilon^{2}$. The exponential term is

$$
\left.-\frac{1}{2}\left(\mathbf{x}-\boldsymbol{\mu}_{A B B}\right)^{T} \Sigma^{-2}\left(\mathbf{x}-\boldsymbol{\mu}_{A B B}\right)=-\frac{1}{2 \sigma^{2}}\left\{\begin{array}{ccc}
x_{1}-\mu_{A} & x_{2}-\mu_{B} & x_{3}-\mu_{B}
\end{array}\right]\left[\begin{array}{ccc}
1+b & b & b \\
b & 1+b & b \\
b & b & 1+b
\end{array}\right]\left[\begin{array}{l}
x_{1}-\mu_{A} \\
x_{2}-\mu_{B} \\
x_{3}-\mu_{B}
\end{array}\right]\right\},
$$

where $b$ is defined in Equation A4. The above equations can be helpful in solving the expression for the psychometric function.

\section{APPENDIX B \\ Derivation of Some Properties of the Coordinate Transformation to Make Observations Independent}

Because $\Sigma^{2}$ is symmetric $\left(\Sigma_{i j}^{2}=\Sigma_{j i}^{2}\right)$, there exists an orthogonal matrix $\Omega$, such that

$$
\Sigma 2 \Omega=\Omega \Lambda
$$

and $\Lambda$ is a diagonal matrix. The geometrical interpretation is that $\Omega$ performs a rotation of the coordinate axes in such a way that in the new coordinate system, observations are not correlated. A possible coordinate transformation (cf. Durlach, Braida, \& Ito, 1986) is given by

$$
\left\{\begin{array}{l}
z_{1}=\frac{1}{\sqrt{m}} \sum_{n=1}^{m} x_{n} \\
z_{j}=\frac{1}{\sqrt{j(j-1)}}\left[j x_{j}-\sum_{n=1}^{j} x_{n}\right]
\end{array}\right.
$$

The new covariance matrix $\Lambda$ then is a diagonal matrix

$$
\Lambda=\sigma^{2}\left[\begin{array}{cccccc}
\varepsilon^{-2} & & & & & \\
& 1 & & & 0 & \\
& & \cdot & & \\
0 & & & \cdot & \\
& & & \cdot & \\
& & & & & 1
\end{array}\right] .
$$

The expected values $\boldsymbol{\mu}_{s}$ transform to $\boldsymbol{\nu}_{s}$, 


$$
\left\{\begin{array}{l}
v_{s 1}=\frac{1}{\sqrt{m}} \sum_{n=1}^{m} \mu_{s n} \\
v_{s j}=\frac{1}{\sqrt{j(j-1)}}\left[j \mu_{s j}-\sum_{n=1}^{j} \mu_{s n}\right]
\end{array}\right.
$$

The transformed density functions can be written as

$$
\begin{aligned}
p d f_{s}(\mathbf{z}) & =\frac{1}{\sqrt{(2 \pi)^{m}|\Lambda|}} \exp \left[-\frac{1}{2}\left(\mathbf{z}-\boldsymbol{\nu}_{s}\right)^{T} \Lambda^{-1}\left(\mathbf{z}-\boldsymbol{\nu}_{s}\right)\right] \\
& =\frac{\varepsilon}{(2 \pi)^{m / 2} \sigma^{m}} \exp \left[-\frac{1}{2 \sigma^{2}}\left\{\varepsilon^{2}\left(z_{1}-v_{s 1}\right)^{2}+\sum_{j=2}^{m}\left(z_{j}-v_{s j}\right)^{2}\right\}\right] .
\end{aligned}
$$

Equation B6 shows that when the transformation is made, (1) the observations become uncorrelated and (2) the common variance $\sigma_{R}^{2}$ is situated in one dimension only.

The inverse functions of Equation $B 2$ are given by

$$
\left\{\begin{array}{l}
x_{1}=\frac{1}{\sqrt{m}} z_{1}-\sum_{n=2}^{m} \frac{1}{\sqrt{n(n-1)}} z_{n} \\
x_{j}=\frac{1}{\sqrt{m}} z_{1}+\frac{j-1}{\sqrt{j(j-1)}} z_{j}-\sum_{n=j+1}^{m} \frac{1}{\sqrt{n(n-1)}} z_{n} . \\
x_{m}=\frac{1}{\sqrt{m}}\left(z_{1}+z_{m} \sqrt{m-1}\right)
\end{array}\right.
$$

The decision boundaries given in Equation 18 now can easily be transformed with the expressions given in Equation B7, which then yield

$$
\begin{aligned}
& \left\{\begin{array}{l}
\frac{k-1}{\sqrt{k(k-1)}} z_{k}+\sum_{n=2}^{k} \frac{1}{\sqrt{n(n-1)}} z_{n}=0 \\
\frac{(m-2) \varepsilon^{2}}{\sqrt{m}}\left(z_{1}-\mu \sqrt{m}\right)-\frac{k}{\sqrt{k-1}} z_{k}+\sum_{n=2}^{m} \frac{1}{\sqrt{n(n-1)}} z_{n}+\sum_{n=k}^{m} \frac{1}{\sqrt{n(n-1)}} z_{n}=0
\end{array} \text { for } 1<k<m .\right. \\
& \left\{\begin{array}{l}
\frac{m-1}{\sqrt{m}} z_{m}+\sum_{n=2}^{m} \frac{1}{\sqrt{n(n-1)}} z_{n}=0 \\
\frac{(m-2) \varepsilon^{2}}{\sqrt{m}}\left(z_{1}-\mu \sqrt{m)}-\frac{m}{\sqrt{m-1}} z_{m}+\sum_{n=2}^{m} \frac{1}{\sqrt{n(n-1)}} z_{n}=0 \quad \text { for } k=m .\right.
\end{array}\right.
\end{aligned}
$$

Although the expression for the boundaries is less simple, a major advantage is achieved, because the density functions are uncorrelated.

(Manuscript received June 10, 1994;

revision accepted for publication May 5, 1995.) 